\title{
Luxación posterior inveterada de hombro asociada a lesión de Hill-Sachs reversa tratada con modificación de la técnica de McLaughlin
}

\author{
Unreduced posterior dislocation of the shoulder with an associated reverse \\ Hill-Sachs injury treated with a modified McLaughlin technique
}

\author{
Ortiz-Díaz LG, * Magallanes-Durán E, * Martínez-Pérez R*
}

Centenario Hospital Miguel Hidalgo, Aguascalientes, Ags.

\begin{abstract}
RESUMEN. Introducción: Las luxaciones posteriores de hombro son raras (2\%) y se asocian a convulsiones, electrocuciones y traumatismos de alta energía. Pueden presentarse asociadas a una lesión de Hill Sachs reversa. Uno de los principios del tratamiento consiste en la transferencia del tendón subescapular hacia el área de lesión o procedimiento de McLaughlin. Caso clínico: Se expone el caso de un masculino con un defecto de Hill-Sachs reverso tratado con una modificación de la técnica original de McLaughlin. Se reportan los resultados funcionales tras 13 meses de la cirugía. Resultados: Actualmente con un Constant Score de 98 puntos. Discusión: La modificación técnica empleada para el tratamiento quirúrgico de la lesión de Hill Sachs reversa presentada en este paciente demostró bueos resultados funcionales con material de bajo costo.
\end{abstract}

Palabras clave: Luxación posterior de hombro, HillSachs reversa, procedimiento de McLaughlin.

\section{Introducción}

La inestabilidad glenohumeral posterior involucra la lesión de cualquiera de las estructuras que conforman el hombro como el labrum, la cápsula, los ligamentos glenohumerales o una lesión ósea intraarticular. ${ }^{1}$ Estas luxaciones son raras, representan $2 \%$ de eventos de inestabilidad de hombro; están frecuentemente asociadas a convulsiones,

\footnotetext{
* Centenario Hospital Miguel Hidalgo. Servicio de Traumatología y Ortopedia. Módulo de cirugía articular. Aguascalientes, Ags.

Dirección para correspondencia:

Dr. Esteban Magallanes Durán

E-mail: esteban_719_6@hotmail.com

Este artículo puede ser consultado en versión completa en: www.medigraphic.com/actaortopedica
}

ABSTRACT. Introduction: Posterior shoulder dislocations are rare (2\%) and are associated with seizures, electrocutions and high-energy trauma. They may be associated with a reverse Hill-Sachs lesion. One of the treatment principles consists of the subscapular tendon transfer to the injury area or McLaughlin procedure. Clinical case: A case of a man with a reverse Hill-Sachs defect treated with a modification of McLaughlin's original technique is presented. The functional results after 13 months of surgery are reported. Results: Currently with a Constant Score of 98 points. Conclusion: The technical modification used for the surgical treatment of the reverse Hill-Sachs lesion presented in this patient demonstrated good functional results with low cost material.

Keywords: Posterior shoulder dislocation, Hill-Sachs reverse, McLaughlin procedure.

electrocuciones y traumatismos de alta energía en pacientes jóvenes. Pueden asociarse a una lesión de Hill-Sachs reversa, la cual resulta de la impactación del rodete glenoideo posterior con la cara anterior de la cabeza humeral. Para su tratamiento se emplea el procedimiento de McLaughlin, así como las modificaciones descritas de la técnica original, la cual consiste en la transferencia del tendón subescapular hacia el defecto. ${ }^{1,2}$

Se presenta el caso de un paciente con un defecto de Hill-Sachs reverso no diagnosticado de forma inicial, tratado seis semanas después con una modificación de la técnica de McLaughlin, se reportan los resultados funcionales tras 13 meses de seguimiento con la escala de Constant Score.

Reporte de caso

Hombre de 54 años de edad, de oficio electricista, acudió al servicio de urgencias por dolor y deformidad de hombro 
derecho de una semana de evolución tras sufrir descarga eléctrica mientras laboraba, lo que provocó dolor agudo así como rigidez y limitación de los arcos de movilidad del hombro.

A la exploración física con arcos de movilidad de hombro derecho: flexión de $80^{\circ}$, abducción de $50^{\circ}$, extensión de $10^{\circ}$, con bloqueo de rotación externa. Se obtiene una radiografía anteroposterior de hombro derecho que muestra incongruencia de articulación glenohumeral y trazo de fractura en el troquín. Se solicitó tomografía axial computarizada simple de hombro, el paciente se presentó a consulta externa de cirugía articular hasta dos semanas después con estudio solicitado.

Las imágenes confirmaron la luxación glenohumeral posterior derecha inveterada y engranada, así como una lesión de Hill-Sachs reversa asociada (Figura 1). Se otorgó tratamiento quirúrgico con reducción abierta y transferencia de subescapular con técnica de McLaughlin modificada tras seis semanas de la lesión.

Técnica quirúrgica: se usó anestesia general y posición de silla de playa, con abordaje deltopectoral, se desinserta el subescapular; se incide la cápsula, con ayuda de un separador de Hohmman se realiza la reducción. Se localiza el fragmento del troquín consolidado con una lesión de Hill-Sachs de $2 \times 2 \mathrm{~cm}$, se hace la limpieza del defecto y se efectúa la transferencia del subescapular, cubriendo lesión de Hill-Sachs reversa con cuatro puntos transóseos sobre el borde anterior del defecto con sutura de poliéster trenzado (Ethibond $^{\circledR} \#$ ), se introduce la aguja primero por el borde articular sacándola por el defecto, después se sutura el tendón para finalmente anudarse dejando el borde del subescapular ${ }^{3}$ dentro del defecto y los nudos sobre el tendón del subescapular (Figura 2). Se afronta por planos y se coloca inmovilizador universal de hombro (Figura 3).

\section{Resultados}

A las dos semanas postquirúrgicas acude a revisión de heridas; se continúa con uso de inmovilizador. A las seis semanas postquirúrgicas se comienza con movimientos pasivos de hombro y ejercicios pendulares de Codman durante dos semanas, alternando uso de inmovilizador de hombro con cojinete abductor (Figura 4).

A las ocho semanas postquirúrgicas presenta arcos de movilidad pasivos flexión de $100^{\circ}$, abducción de $100^{\circ}$, rotación externa $15^{\circ}$. Se inician movimientos activos para mejoría de arcos de movilidad, después de 12 semanas de la cirugía se reevalúan arcos de movilidad activos, alcanza: flexión $170^{\circ}$ abducción $170^{\circ}$, rotación externa $45^{\circ}$, rotación interna S1, extensión $30^{\circ}$. A los 13 meses de seguimiento alcanza flexión
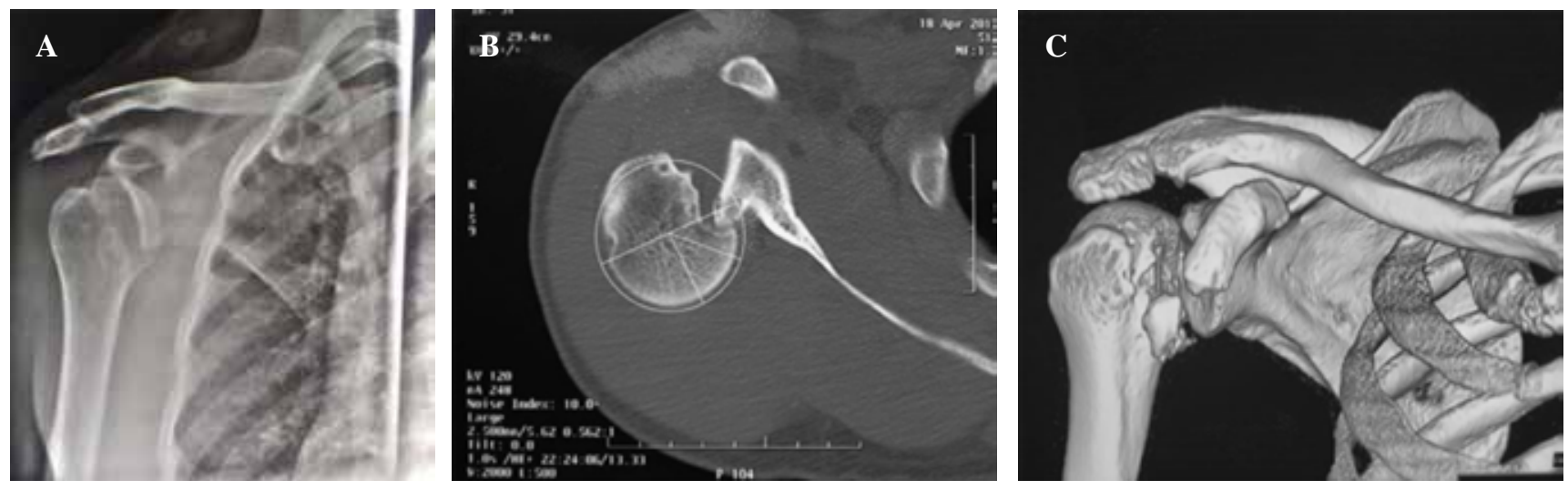

Figura 1: A) Radiografía anteroposterior verdadera de hombro, se observa incongruencia de articulación glenohumeral. B) Corte axial de tomografía simple de hombro, se observa impactación de rodete glenoideo posterior sobre la superficie anterior de cabeza humeral $(<25 \%)$. C) Reconstrucción de tomografía simple de hombro.
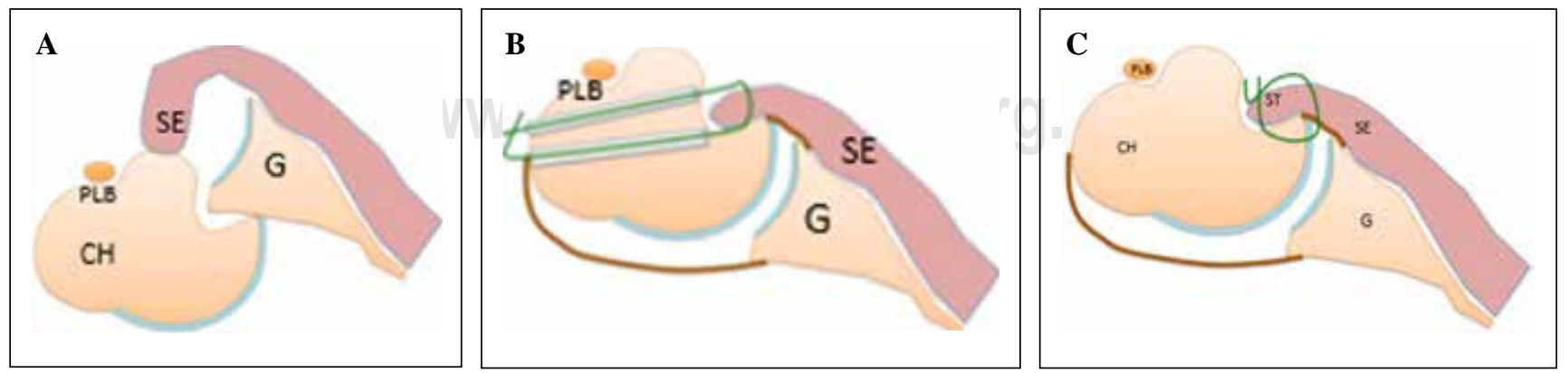

Figura 2: A) Esquema de luxación posterior con lesión de Hill-Sachs. B) Técnica de McLaughlin. C) Modificación de técnica de McLaughlin.

$\mathrm{CH}=$ cabeza humeral, $\mathrm{G}=$ glenoides, $\mathrm{SE}=$ subescapular, $\mathrm{ST}=$ sutura transósea, PLB = porción larga del tendón del bíceps. 

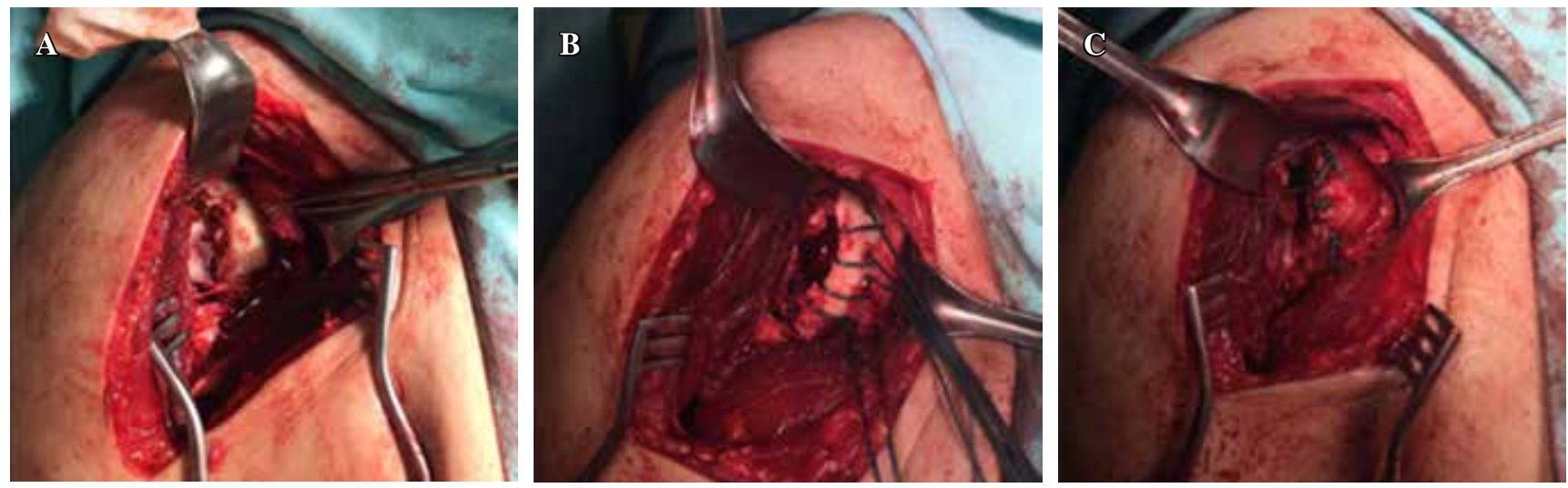

Figura 3: A) Lesión de Hill-Sachs en superficie anterior de cabeza humeral a través de abordaje quirúrgico. B) Avance de tendón subescapular sobre lesión de Hill-Sachs, se observan suturas de poliéster trenzado 5-0. C) Recubrimiento de lesión de Hill-Sachs reversa, se aísla defecto quedando extraarticular.

\begin{tabular}{|c|c|c|c|c|}
\hline & \multirow{2}{*}{$\begin{array}{c}\text { Prequirúrgico } \\
\text { Inicial }\end{array}$} & \multicolumn{3}{|c|}{ Postquirúrgico } \\
\hline & & 8 semanas & 12 semanas & 13 meses \\
\hline Flexión & $80^{\circ}$ & $100^{\circ}$ & $170^{\circ}$ & $180^{\circ}$ \\
\hline Abducción & $50^{\circ}$ & $100^{\circ}$ & $170^{\circ}$ & $180^{\circ}$ \\
\hline Extensión & $10^{\circ}$ & $10^{\circ}$ & $30^{\circ}$ & $30^{\circ}$ \\
\hline Rotación externa & - & $15^{\circ}$ & $45^{\circ}$ & $70^{\circ}$ \\
\hline Rotación interna & - & - & 51 & $\mathrm{~T} 12$ \\
\hline
\end{tabular}

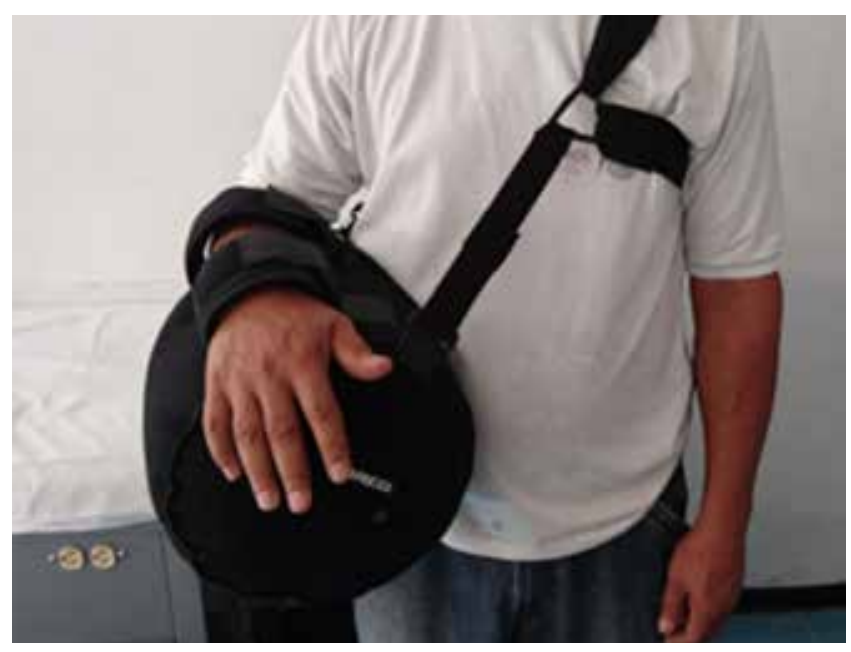

Figura 4: Aparato abductor empleado en la rehabilitación del paciente durante seis semanas.

de $180^{\circ}$, abducción $180^{\circ}$, rotación interna alcanza el nivel de $\mathrm{T} 12$, rotación externa de $70^{\circ}$ (Figura 5), con un puntaje de Constant Score de 98 sobre 100 (dolor 25; actividades cotidianas 20; arcos de movilidad 38; fuerza 25) (Tabla 1).

\section{Discusión}

La luxación posterior de hombro se ha descrito de fácil omisión diagnóstica. De acuerdo a la serie reportada por
Drudvic, ${ }^{4} 21$ de 24 pacientes no fueron diagnosticados en su valoración inicial. Hay distintas alternativas quirúrgicas para su tratamiento, debido a que no existe una lesión única que explique la inestabilidad. Los distintos métodos consisten en la reparación de defectos óseos y de tejidos blandos a través de abordajes abiertos, artroscópicos o combinados. En el caso descrito, existe compromiso de menos de 50\% de la superficie articular de la cabeza humeral, por lo que se prefirió la transferencia de inserción del tendón subescapular sobre el sitio de lesión. ${ }^{5}$ De acuerdo a la escala de Constant Score, los resultados funcionales obtenidos son comparables a los reportados en la literatura por Kokkalis y colaboradores, ${ }^{6}$ quienes describieron el tratamiento de seis hombros con técnica modificada de McLaughlin con resultados funcionales, con un puntaje de 84 y un restablecimiento casi completo de los arcos de movilidad del hombro; esta serie empleó la modificación de Neer. Los resultados funcionales finales aquí presentados no se vieron afectados por la modificación del método de inserción del tendón en la cabeza humeral y el inicio temprano de los movimientos pasivos en este paciente.

La modificación técnica con una sutura transósea empleada para el tratamiento quirúrgico de la lesión de HillSachs reversa en lesiones menores de $25 \%$ de la superficie articular tiene buenos resultados funcionales, sin necesidad de colocación de injerto óseo; se facilitó la ejecución de la técnica al no realizar túneles transóseos así como disminución del costo al no usar materiales extra. 

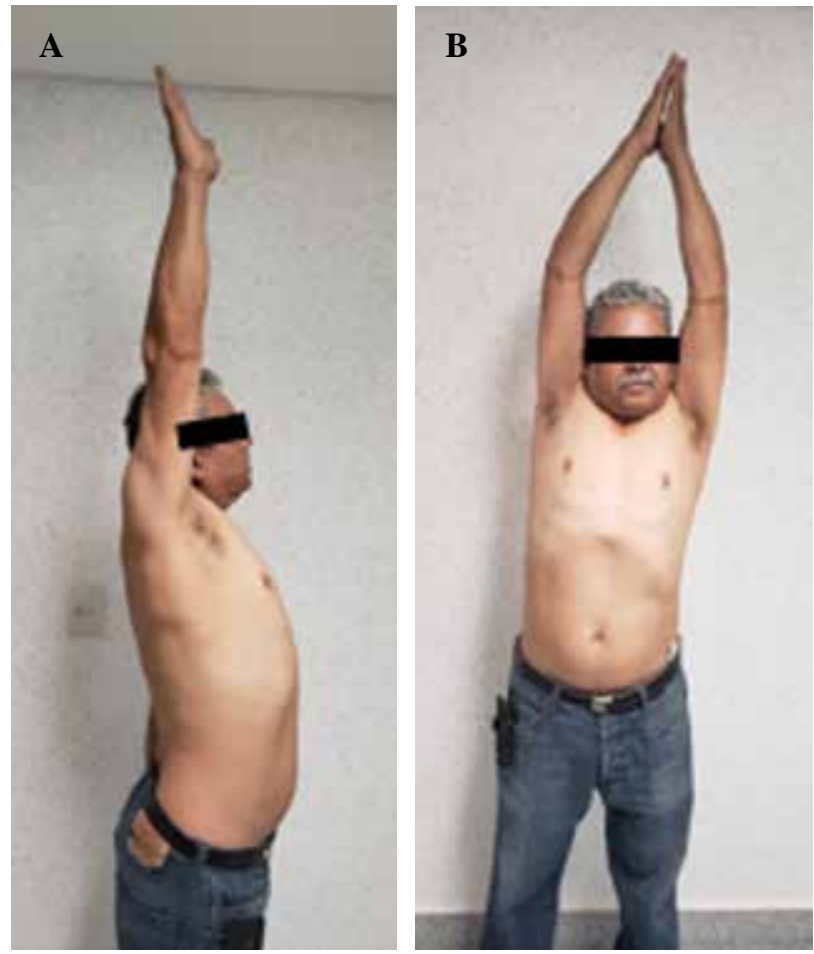

Bibliografía

1. Antosh IJ, Tokish JM, Owens BD. Posterior Shoulder Instability: current surgical management. Sports Health. 2016; 8(6): 520-6.

2. McLaughlin HL. Posterior dislocation of the shoulder. $J$ Bone Joint Surg. 1952; 34A(3): 548-90.

3. Konda SR, Fisher N, Gage M, Egol K. Posterior fracture dislocation of the shoulder: a modified McLaughlin Procedure. J Orthop Trauma. 2017; 31(Suppl 3): S36-7.

4. Djurdjevic D. Unrecognized posterior dislocation of the shoulder. Masters Thesis, Zagreb, 2003.
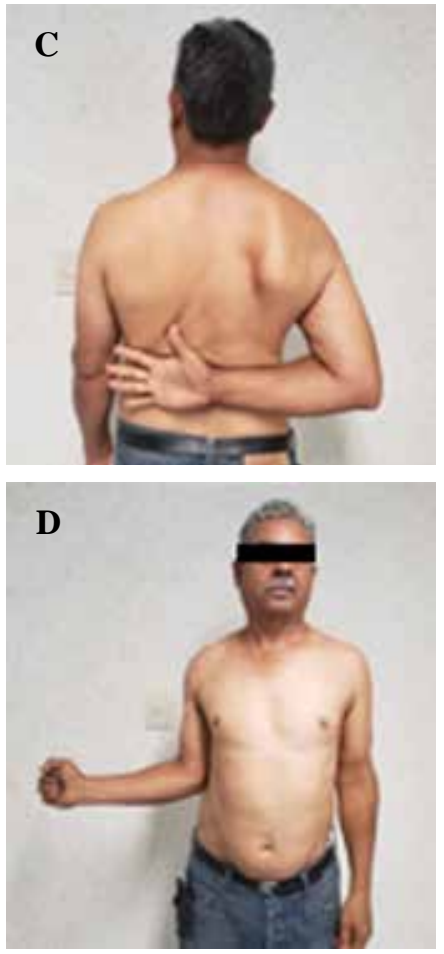

Figura 5:

Evaluación funcional tras 13 meses. A) Flexión activa máxima conseguida $\left(180^{\circ}\right)$. B) Abducción activa máxima $\left(180^{\circ}\right)$. C) Rotación interna activa (T12). D) Rotación externa activa conseguida $\left(70^{\circ}\right)$.

5. Cicak N. Posterior dislocation of the shoulder. J Bone Joint Surg. 2004; 86(3): 324-32.

6. Kokkalis ZT, Mavrogenis AF, Ballas EG, Papanastasiou J, Papagelopoulos PJ. Modified McLaughlin technique for neglected locked posterior dislocation of the shoulder. Orthopedics. 2013; 36(7): e912-6.

Conflicto de intereses: Los autores declaran que no existe conflicto de intereses. 